\title{
BMJ Open High reproducibility of adenosine stress cardiac MR myocardial perfusion imaging in patients with non-ischaemic dilated cardiomyopathy
}

\author{
Mark A Lawson, ${ }^{1}$ Susan P Bell, ${ }^{1}$ Douglas W Adkisson, ${ }^{1}$ Li Wang, ${ }^{2}$ Henry Ooi, ${ }^{1,3}$ \\ Douglas B Sawyer, ${ }^{1}$ Marvin W Kronenberg ${ }^{1,3}$
}

To cite: Lawson MA, Bell SP, Adkisson DW, et al. High reproducibility of adenosine stress cardiac MR myocardial perfusion imaging in patients with non-ischaemic dilated cardiomyopathy. BMJ Open 2014;4:e005984. doi:10.1136/bmjopen-2014005984

- Prepublication history for this paper is available online. To view these files please visit the journal online (http://dx.doi.org/10.1136/ bmjopen-2014-005984).

Received 26 June 2014 Revised 20 November 2014 Accepted 21 November 2014

CrossMark

\footnotetext{
${ }^{1}$ Division of Cardiovascular Medicine, Vanderbilt University School of Medicine, Nashville, Tennessee, USA

2Department of Biostatistics, Vanderbilt University Medical Center, Nashville, Tennessee, USA

${ }^{3}$ Cardiology Section, Department of Veterans Affairs Tennessee Valley Healthcare System, Nashville, Tennessee, USA

Correspondence to Dr Marvin W Kronenberg; marvin.w.kronenberg@ vanderbilt.edu
}

\section{ABSTRACT}

Objective: To evaluate the reproducibility of first-pass contrast-enhanced cardiac MR (CMR) myocardial perfusion imaging in patients with non-ischaemic dilated cardiomyopathy (NIDCM).

Design: Prospective observational study.

Setting: Single centre, tertiary care hospital.

Participants: 6 outpatient participants with NIDCM.

Outcome: Reproducibility of semiquantitative myocardial perfusion analysis by CMR.

Method: 6 patients with NIDCM were studied twice using first-pass of contrast transit through the left ventricular (LV) myocardium with a saturation-recovery gradient echo sequence at rest and during adenosineinduced hyperaemia. The anterior wall was divided into endocardial (Endo) and epicardial (Epi) segments. The Myocardial Perfusion Index (MPI) was calculated as the myocardial signal augmentation rate normalised to the LV cavity rate. The Myocardial Perfusion Reserve Index (MPRI) was calculated as hyperaemic/resting MPI.

Results: Between study 1 and 2, median MPI was similar for resting Endo (0.076 vs 0.077 ), hyperaemic Endo (0.143 vs 0.143 ), resting Epi ( 0.073 vs 0.074$)$, and hyperaemic Epi (0.135 vs 0.134$)$. Median MPRI was similar for Endo (1.84 vs 1.87) and Epi (1.90 vs 2.00). Combining Endo and Epi MPI ( $N=12)$, there was excellent agreement between Study 1 and 2 for resting MPI ( $r=0.998$, intraclass correlation coefficient (ICC) 0.998, coefficients of variation (CoV) $1.4 \%$ ), hyperaemic MPI ( $\mathrm{r}=0.979$, ICC 0.963 , CoV 3.3\%) and MPRI ( $r=0.989$, ICC 0.94, CoV 3.8\%).

Conclusions: Resting and hyperaemic myocardial perfusion using a normalised upslope analysis during adenosine CMR is a highly reproducible technique in patients with NIDCM.

Trial registration number: Clinical Trials.Gov ID NCT00574119.

\section{INTRODUCTION}

The technique for evaluating myocardial perfusion using cardiovascular MR (CMR) imaging is based on imaging the first-pass

\section{Strengths and limitations of this study}

- Excellent reproducibility and agreement of firstpass, contrast-enhanced cardiac MR (CMR) myocardial perfusion imaging in a cohort of patients undergoing treatment for non-ischaemic dilated cardiomyopathy.

- Enhanced spatial resolution of CMR is a potentially valuable tool to evaluate how endocardial hypoperfusion contributes to the pathophysiology of heart failure and whether heart failure treatment may favourably alter perfusion.

- To date, only three studies have published on reproducibility data using this CMR technique in humans (combined total of 28 normal or "low risk' participants and 19 patients with coronary artery disease).

- Small sample size may limit the statistical power of the findings; however, despite the small sample size, the reproducibility was excellent and the statistical power was very strong.

transit of contrast through the myocardium following its rapid intravenous bolus injection. ${ }^{1}$ The first-pass technique is often performed under resting conditions and during the peak vasodilatory hyperaemic effect of a continuous intravenous infusion of adenosine. The clinical utility and diagnostic accuracy of adenosine stress CMR perfusion imaging for detecting obstructive epicardial coronary artery disease has been demonstrated in several large multicenter trials and performs favourably with other non-invasive diagnostic testing. ${ }^{2-4}$ Myocardial perfusion abnormalities are also present in other conditions, such as endothelial dysfunction (so-called 'syndrome X') using $\mathrm{CMR}^{5}{ }^{6}$ and non-ischaemic dilated cardiomyopathy (NIDCM) using positron emission tomography. ${ }^{7} 8$

Endocardial hypoperfusion during vasodilator stress has been observed in animal models of heart failure, ${ }^{9-11}$ and a blunted 
coronary vasodilatory reserve has been thought to contribute to the condition of 'energy starvation' in heart failure in humans. ${ }^{12}{ }^{13}$ Based on the enhanced spatial resolution of CMR, we have shown that subendocardial perfusion is reduced and perfusion reserve is blunted in patients with NIDCM. ${ }^{14}$ The reproducibility of the CMR technique has been studied only in a small number of normal participants and patients with coronary artery disease $\mathrm{e}^{15-17}$ but has not been previously reported in patients with NIDCM. Accordingly, we evaluated the reproducibility of first-pass contrast-enhanced CMR to assess indices of endocardial and epicardial perfusion performed in a cohort of treated patients with NIDCM under resting conditions and during a continuous intravenous adenosine infusion.

\section{METHODS}

This study was conducted as part of a prospective interventional trial investigating the effects of a mineralocorticoid receptor antagonist, spironolactone, in 12 patients with NIDCM. To evaluate the reproducibility of adenosine stress CMR, half of these patients $(\mathrm{N}=6)$ underwent two identical CMR examinations while on stable medical therapy for heart failure using $\beta$-adrenergic blockade, either an angiotensin converting enzyme (ACE) inhibiting or angiotensin receptor blocking drug, and spironolactone. The testing of reproducibility was prospectively evaluated in the final half $(\mathrm{N}=6)$ of the patients enrolled. Patients with newly diagnosed NIDCM were recruited. Inclusion criteria for eligible participants at enrolment were: age between 18 and 80 years, New York Heart Association Functional Class II-IV, a left ventricular (LV) ejection fraction (LVEF) of $\leq 35 \%$ by echocardiography and a serum potassium level $<5.0 \mathrm{mg} / \mathrm{dL}$ while on a minimum of 3 months of stable medical therapy for heart failure including $\beta$-adrenergic blockade and either an ACE inhibiting or angiotensin receptor blocking drug. Individuals with a need for an implantable cardioverter-defibrillator or $>50 \%$ stenosis in a major epicardial artery at angiography were excluded. Further exclusion criteria included severe chronic obstructive airway disease precluding adenosine use, creatinine $>2.5 \mathrm{mg} / \mathrm{dL}$, glomerular filtration rate $<30 \mathrm{~mL} / \mathrm{min} /$ $1.73 \mathrm{~m}^{2}$, uncontrolled atrial fibrillation, spironolactone therapy at recruitment and physician preference. Patients were instructed not to consume caffeinated or decaffeinated beverages for $24 \mathrm{~h}$, prior to the CMR study. All patients provided a written informed consent.

\section{Cardiac MRI}

Cardiac MRI was performed using a commercially available 1.5 T Siemens Magnetom Avanto scanner (Erlangen, Germany). Patients were scanned using a phased-array torso receiver coil. The imaging protocol consisted of three parts: (1) cine imaging for ventricular volume and function, (2) first-pass contrast-enhanced myocardial perfusion imaging during maximal adenosine-induced coronary vasodilation and under resting conditions, and (3) myocardial late gadolinium enhancement imaging.

For the perfusion study, adenosine $(140 \mu \mathrm{g} / \mathrm{kg} / \mathrm{min})$ was infused intravenously over 4-6 min using an infusion pump (Continuum, Medrad, Warrendale, Pennsylvania, USA). Approximately $4 \mathrm{~min}$ into the adenosine infusion, $0.1 \mathrm{mmol} / \mathrm{kg}$ gadolinium-diethylenetriamine penta-acetic acid (Gd-DTPA) (Magnevist, Bayer HealthCare Pharmaceuticals, Wayne, Indiana, USA) was injected intravenously into an antecubital vein (9 right, 3 left) at $5 \mathrm{~mL} / \mathrm{s}$ using a power injector (Spectris Solaris, Medrad, Warrendale, Pennsylvania, USA), followed by a $15 \mathrm{~mL}$ saline flush. First-pass imaging of the wash-in of Gd-DTPA through the LV myocardium was performed in three short-axis imaging planes positioned in mid myocardial segments of the LV using a saturation-recovery turbo fast low-angle shot (FLASH) gradient echo sequence. All three short-axis images were acquired with each R-R interval over 50 consecutive heart beats starting with the injection of Gd-DTPA in order to capture the initial wash-in of Gd-DTPA through the myocardium. Following a 5-min delay, contrast-enhanced first-pass imaging was repeated under resting conditions with a second intravenous injection of $0.1 \mathrm{mmol} / \mathrm{kg}$ Gd-DTPA. All images were corrected for surface coil intensity variation using a normalisation filter. The typical acquisition parameters for perfusion images were: field of view (FOV) $300 \times 340 \mathrm{~mm}$, matrix $156 \times 192$, slice thickness (SL) $8 \mathrm{~mm}$, flip angle (FA) $12^{\circ}$, echo time (TE) $1.1 \mathrm{~ms}$, bandwidth (BW) $930 \mathrm{~Hz} /$ pixel, repetition time (TR) $180 \mathrm{~ms}$ and a variable trigger delay depending on heart rate to acquire data when possible in diastole. Parallel imaging was employed using the generalised autocalibrating partially parallel acquisition (GRAPPA) technique with an acceleration factor of 2. Blood pressure and heart rate were recorded at baseline and every minute during the adenosine infusion.

The LV volume, EF and mass were calculated from manually traced endocardial and epicardial end-diastolic and end-systolic contours from a stack of contiguous short-axis images that covered the LV from the apex to the base of the heart. These variables were calculated on a Leonardo workstation using Argus software, V.B17 (Siemens, Erlangen, Germany).

Myocardial enhancement occurred during the transit of Gd-DTPA through the heart, and the Myocardial Perfusion Index (MPI) was calculated for the endocardial and epicardial segments of the anterior LV wall as illustrated in figure 1 . The endocardial and epicardial borders were semiautomatically drawn with manual editing (to exclude contamination by blood pool or epicardial fat) by one of the authors (DWA) using the analysis software (CMRTools, London, UK). The accuracy of the drawing was verified in a blinded fashion on randomly presented studies by a second author (MAL) with only a few minor edits required. No reference was made to Study 1 when processing Study 2. The anterior wall was selected for analysis to: (1) avoid the so-called 'dark rim' artefact that sometimes is encountered in the 

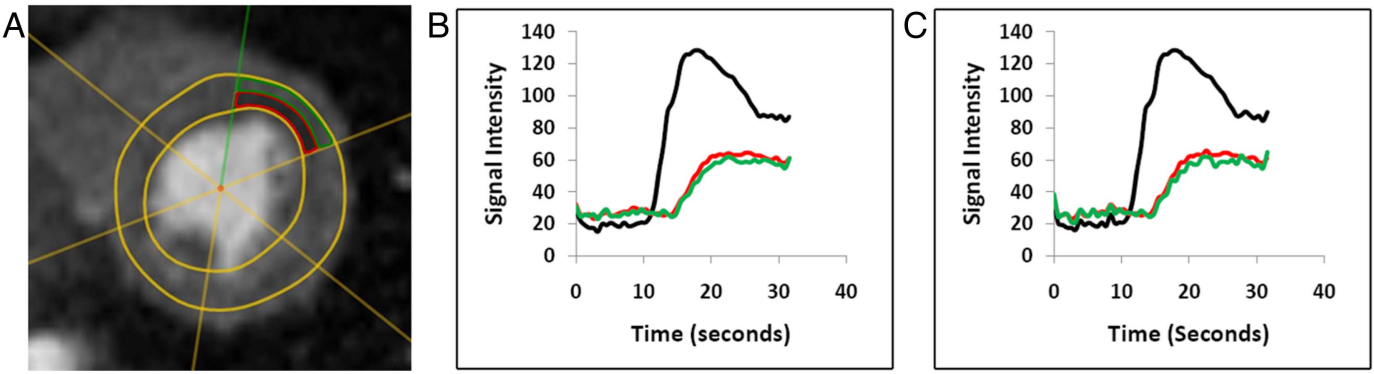

Figure 1 Myocardial perfusion image and analysis. Representative short-axis myocardial perfusion image of a patient segmented into endocardial (red) and epicardial (green) regions (A). Signal intensity-time curves of left ventricular blood pool (black line), anterior wall endocardium (red line) and epicardium (green line) constructed during the first-pass wash-in of contrast from Study 1 (B) and Study 2 (C).

septum and (2) avoid trabeculations along the lateral and inferior LV walls that can be particularly prominent in patients with dilated left ventricles. The anterior wall is generally considered to be the region with the fewest artefacts on CMR analysis. This proved to be the case in our study also. When we examined our cohort of six patients, the changes in MPI and MPRI were not limited to the anterior wall. The average MPRI for all segments showed similar significant results in five of six patients, but with obvious inferior wall artefact in one patient. Thus, to achieve the most valid statistical analysis, we utilised the anterior segment which had no imaging artefacts. MPI was calculated using software as the maximum slope of the time-intensity curve of myocardial enhancement, according to the following equation:

$$
\text { MPI }=\frac{\text { Maximal slope }_{\text {myocardium }}}{\text { Maximal slope }_{\mathrm{LV} \text { cavity }}}
$$

This yielded a normalised upslope of myocardial enhancement that produced a quantitative estimate of myocardial enhancement among the participants as previously described. ${ }^{18-20}$ MPI was calculated for the endocardium and epicardium of a mid-ventricular slice under resting conditions and during adenosine-induced hyperaemia in both studies in all six patients. This yielded a total of 24 measurements of the MPI. A Myocardial Perfusion Reserve Index (MPRI) was also calculated, according to the following equation:

$$
\mathrm{MPRI}=\frac{\mathrm{MPI}_{\text {stress }}}{\mathrm{MPI}_{\mathrm{rest}}}
$$

\section{Statistical analysis}

Linear regression, intraclass correlation coefficient (ICC) and coefficients of variation $\left(\mathrm{CoV}_{1}\right.$ and $\left.\mathrm{CoV}_{2}\right)$ were used to assess the agreement between MPI measurements. The ICG and CoV were calculated for MPRI as well. The ICC was calculated as the ratio of subject variance to the total variance of the cohort. $\mathrm{CoV}_{1}$ was calculated as the ratio of the SD to the mean value of MPI and MPRI, multiplied by $100 . \mathrm{CoV}_{2}$ was calculated as the SD of the differences between Study 1 and 2 divided by the mean and multiplied by 100 . This definition of $\mathrm{CoV}_{2}$ was selected in order to compare our results with previous published reports which used this CoV definition. ${ }^{15-17}$ Study 1 and 2 measurements were also compared using Bland-Altman plots. The limits of agreement were calculated as the average difference \pm 1.96 SD. All data were analysed on SPSS V.18 (SPSS Inc., Chicago, Illinois, USA) and the statistical programming language R V.2.15.1 (R Development Core Team, Vienna, Austria).

\section{RESULTS}

\section{Patient characteristics and adenosine-induced}

\section{haemodynamic changes}

Two patients were men and four were women, ranging in age from 27 to 63 years (median 52 years). A cardiac catheterisation had been performed on all six patients prior to enrolment. No coronary artery stenosis $>50 \%$ was found on coronary angiography. The mean LVEF on the screening echocardiogram before heart failure treatment was $24 \pm 7.7 \%$ (mean \pm 1 SD). The mean LVEF by CMR on heart failure treatment had increased to 48 $\pm 5 \%$. The LV end-diastolic volume averaged $143 \pm 18 \mathrm{~mL}$, LV end-systolic volume $74 \pm 16 \mathrm{~mL}$ and the LV mass 144 $\pm 18 \mathrm{~g}$. The blood pressure and heart rate measured under resting conditions and during the peak hyperaemic effect of adenosine are given in table 1 .

There were no statistically significant differences in the haemodynamic measurements between the two CMR studies. All patients completed the adenosine infusion. All patients were in sinus rhythm, and no arrhythmias were observed during image acquisition.

\section{Interstudy MPI measurements}

Figure 1 illustrates a representative segmented, stillframe short-axis CMR perfusion image and timeintensity curves from Study 1 and 2 in the same patient. Table 2 summarises endocardial and epicardial MPI under resting conditions and during adenosine-induced hyperaemia.

Endocardial and epicardial MPI acquired under resting conditions from the two studies are shown in 
Table 1 Haemodynamic characteristics

\begin{tabular}{|c|c|c|c|}
\hline & Study 1 & Study 2 & p Value \\
\hline \multicolumn{4}{|l|}{ Resting } \\
\hline HR (bpm) & $73 \pm 5$ & $75 \pm 4$ & 0.528 \\
\hline $\mathrm{SBP}(\mathrm{mm} \mathrm{Hg})$ & $124 \pm 13$ & $120 \pm 14$ & 0.528 \\
\hline $\mathrm{DBP}(\mathrm{mm} \mathrm{Hg})$ & $74 \pm 11$ & $72 \pm 12$ & 0.600 \\
\hline RPP (bpm-mm Hg) & $9190 \pm 1205$ & $9060 \pm 1117$ & 0.600 \\
\hline \multicolumn{4}{|c|}{ Adenosine-induced hyperaemia } \\
\hline HR (bpm) & $89 \pm 9$ & $94 \pm 11$ & 0.461 \\
\hline $\mathrm{SBP}(\mathrm{mm} \mathrm{Hg})$ & $125 \pm 13$ & $115 \pm 22$ & 0.075 \\
\hline $\mathrm{DBP}(\mathrm{mm} \mathrm{Hg})$ & $74 \pm 8$ & $74 \pm 8$ & 0.293 \\
\hline RPP (bpm-mm Hg) & $11253 \pm 1729$ & $10752 \pm 1176$ & 0.345 \\
\hline
\end{tabular}

figure 2. The median resting endocardial MPI was 0.076 (IOR 0.066-0.085) for Study 1 and 0.077 (IQR 0.0670.084 ) for Study 2. The median resting epicardial MPI was 0.072 (IQR 0.056-0.081) for Study 1 and 0.074 (IQR $0.056-0.081$ ) for Study 2. Similarly, endocardial and epicardial MPI from the two studies acquired during adenosine infusion are shown in figure 3 . The median hyperaemic endocardial MPI was 0.143 (IQR $0.121-0.156$ ) for Study 1 and 0.143 (IQR 0.120-0.159) for Study 2. The median hyperaemic epicardial MPI was 0.134 (IQR 0.120-0.152) for Study 1 and 0.134 (IQR $0.124-0.158)$ for Study 2.

\section{Interstudy myocardial perfusion reserve measurements}

The MPRI results are tabulated in table 2 and shown in figure 4 . The median endocardial MPRI was 1.84 (IRQ 1.78-1.96) for Study 1 and 1.78 (IQR 1.81-1.95) for Study 2. The median epicardial MPRI was 1.9 (IQR 1.82.2) for Study 1 and 2.0 (IQR 1.8-2.1) for Study 2.

\section{Agreement analysis}

The median time between the two studies was 14 days (range 1-150 days, IQR 1-60 days). Figures 2A and 3A depict excellent correlation between Study 1 and 2 under resting conditions $(r=0.99)$ and during adenosine-induced hyperaemia $(\mathrm{r}=0.98)$. Table 3 shows excellent agreement in MPI and MPRI between Study 1 and 2.
When combining endocardial and epicardial segments $(\mathrm{N}=12)$, the ICC was 0.998 under resting conditions and 0.963 during adenosine-induced hyperaemia. The $\mathrm{CoV}_{1}$ for resting MPI for Study 1 and 2 was $20.2 \%$ and $20.3 \%$, respectively. The $\mathrm{CoV}_{1}$ for hyperaemic MPI for Study 1 and 2 was $13.9 \%$ and $14.1 \%$, respectively. The $\mathrm{CoV}_{2}$ for MPI under resting conditions was $1.4 \%$ and during adenosine-induced hyperaemia was $3.3 \%$. The $\mathrm{CoV}_{2}$ for MPRI was $3.8 \%$. Good agreement was also demonstrated in the Bland-Altman plots (figures 2B, 3B and 4B) which demonstrate very small differences between the two MPI measurements and MPRI as compared to the size of the measurement. No bias had been observed.

\section{DISCUSSION}

This study demonstrates that there is excellent agreement and reproducibility of the first-pass, contrast-enhanced CMR perfusion technique for measuring MPI in patients with NIDCM. Little was known previously about the reproducibility of such measurements in this patient population. Furthermore, a high level of agreement is maintained even after the myocardium is divided into endocardial and epicardial regions. Thus, this technique should be useful for detecting changes in endocardial perfusion in NIDCM and is a potentially valuable tool for the evaluation of endocardial hypoperfusion and the condition of 'energy starvation' in heart failure.

Table 2 Comparison of MPI and MPRI between Study 1 and 2

\begin{tabular}{|c|c|c|c|c|c|c|}
\hline & \multicolumn{3}{|l|}{ Study 1} & \multicolumn{3}{|l|}{ Study 2} \\
\hline & Median & Range & Mean \pm SD & Median & Range & Mean $\pm S D$ \\
\hline Endo (rest) & 0.076 & $0.056-0.094$ & $(0.075 \pm 0.013)$ & 0.077 & $0.056-0.095$ & $(0.076 \pm 0.013)$ \\
\hline Epi (rest) & 0.073 & $0.042-0.093$ & $(0.070 \pm 0.071)$ & 0.074 & $0.042-0.093$ & $(0.070 \pm 0.017)$ \\
\hline Endo (hyperaemia) & 0.143 & $0.112-0.161$ & $(0.139 \pm 0.019)$ & 0.143 & $0.118-0.167$ & $(0.142 \pm 0.019)$ \\
\hline Epi (hyperaemia) & 0.135 & $0.100-0.161$ & $(0.135 \pm 0.021)$ & 0.134 & $0.107-0.169$ & $(0.138 \pm 0.021)$ \\
\hline Endo MPRI & 1.84 & $1.72-2.01$ & $(1.86 \pm 0.12)$ & 1.87 & $1.72-2.11$ & $(1.89 \pm 0.14)$ \\
\hline Epi MPRI & 1.90 & $1.67-2.40$ & $(2.00 \pm 0.30)$ & 2.00 & $1.71-2.55$ & $(2.00 \pm 0.30)$ \\
\hline
\end{tabular}

Endo, endocardial; Epi, epicardial; MPI, Myocardial Perfusion Index; MPRI, Myocardial Perfusion Reserve Index. 

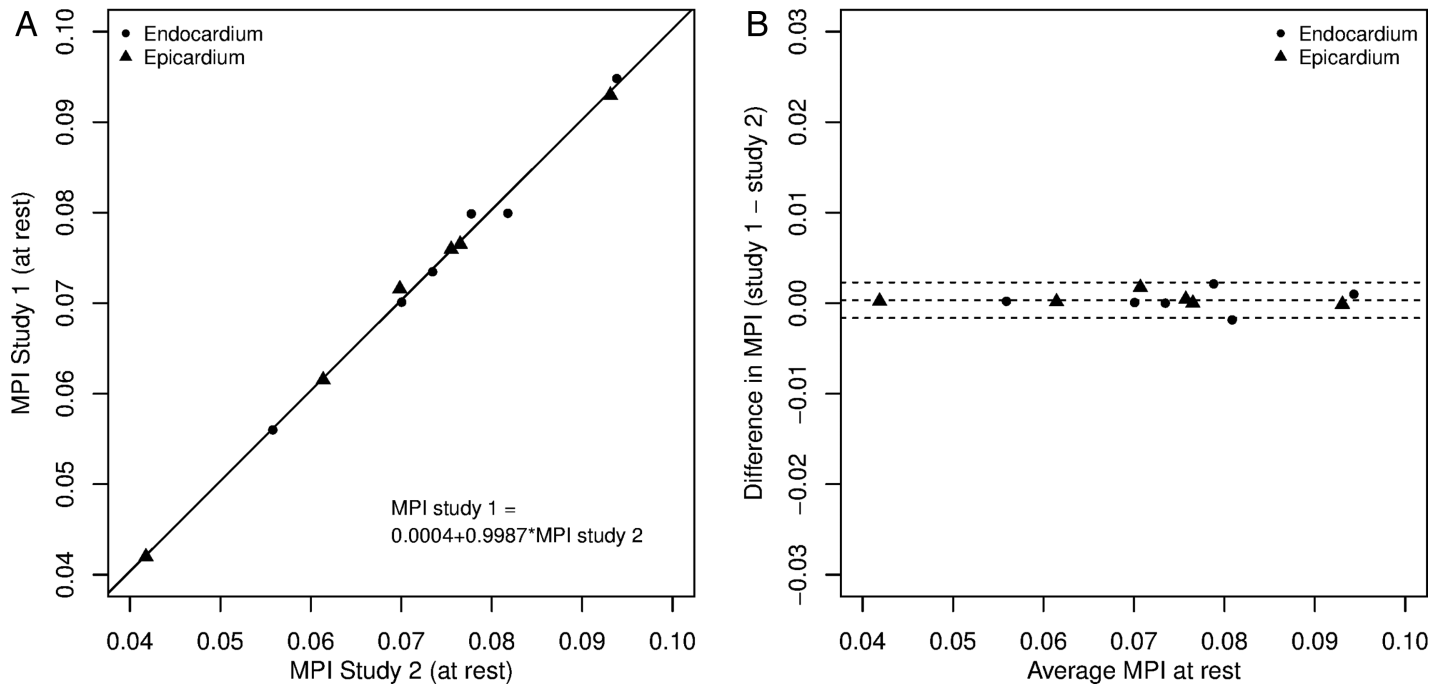

Figure 2 Agreement of resting Myocardial Perfusion Index (MPI) between Study 1 and 2. Linear regression graph (A) and Bland-Altman plot (B) of endocardial (circles) and epicardial (triangles) MPI measured under resting conditions.

Reliability is the reproducibility of a measurement when it is randomly repeated on the same study subject and can be described by several statistical analysis methods. CoV is an index of a test's performance consistency and measures the distribution or dispersion of measurements around the mean. Our $\mathrm{CoV}_{1}$ analysis showed a variation of MPI up to $20 \%$ around the mean MPI for each study subset (either Study 1 or 2); however, the variation in the difference between Study 1 and 2, or $\mathrm{CoV}_{2}$, was $<4 \%$. CoV is impacted by scale and the number of observations (which is indirectly included in the denominator for $\mathrm{CoV}$ ). Since our mean MPI scale is very low, $\mathrm{CoV}$ is exquisitely sensitive to small changes in the mean, and therefore, is not an ideal statistical tool and does not accurately measure reliability. ${ }^{21}$ Therefore, we employed ICC as an index of reliability to assess the consistency of measurements made over multiple

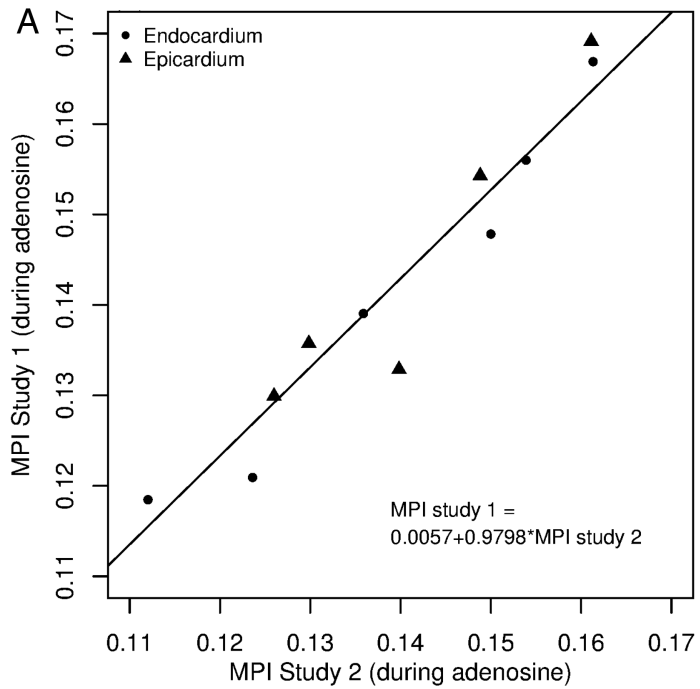

observations. ICC is a calculated value using the formula, ICC $=1-$ (percent variation) within repeated measures relative to the total variation of the measures. The ICG showed excellent reliability of this CMR technique to measure MPI with $<4 \%$ variation across all measurements. Since some lack of agreement between measurements is inevitable, the Bland-Altman plot graphically depicts the amount of disagreement between measurements (via their differences). Our MPI measurements showed excellent agreement with small differences between measurements such that there was a tight range of the limits of agreement about the mean of the differences (which was close to 0 ). There was no systematic variation across the range of measurements.

Published reproducibility data using the upslope method are limited and have been studied in small patient populations. Three studies have reported

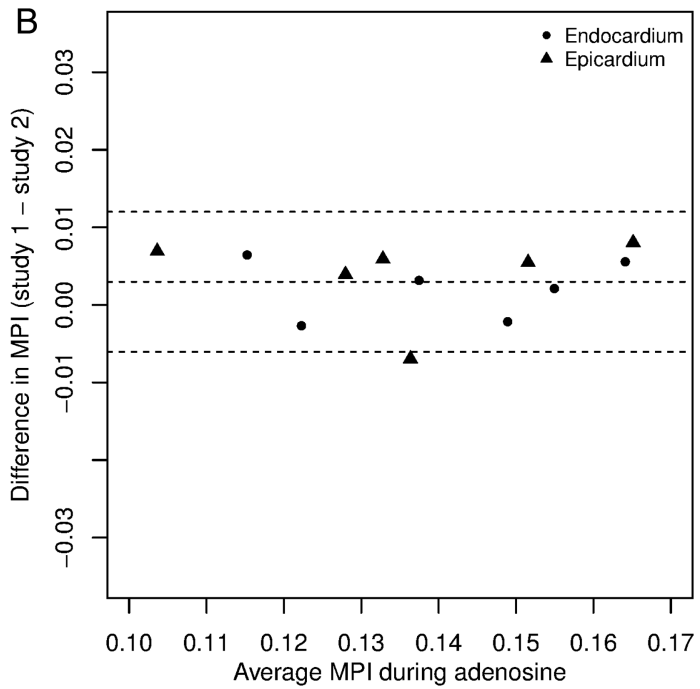

Figure 3 Agreement of hyperemic Myocardial Perfusion Index (MPI) between Study 1 and 2. Linear regression graph (A) and Bland-Altman plot (B) of endocardial (circles) and epicardial (triangles) MPI measured during adenosine-induced hyperaemia. 

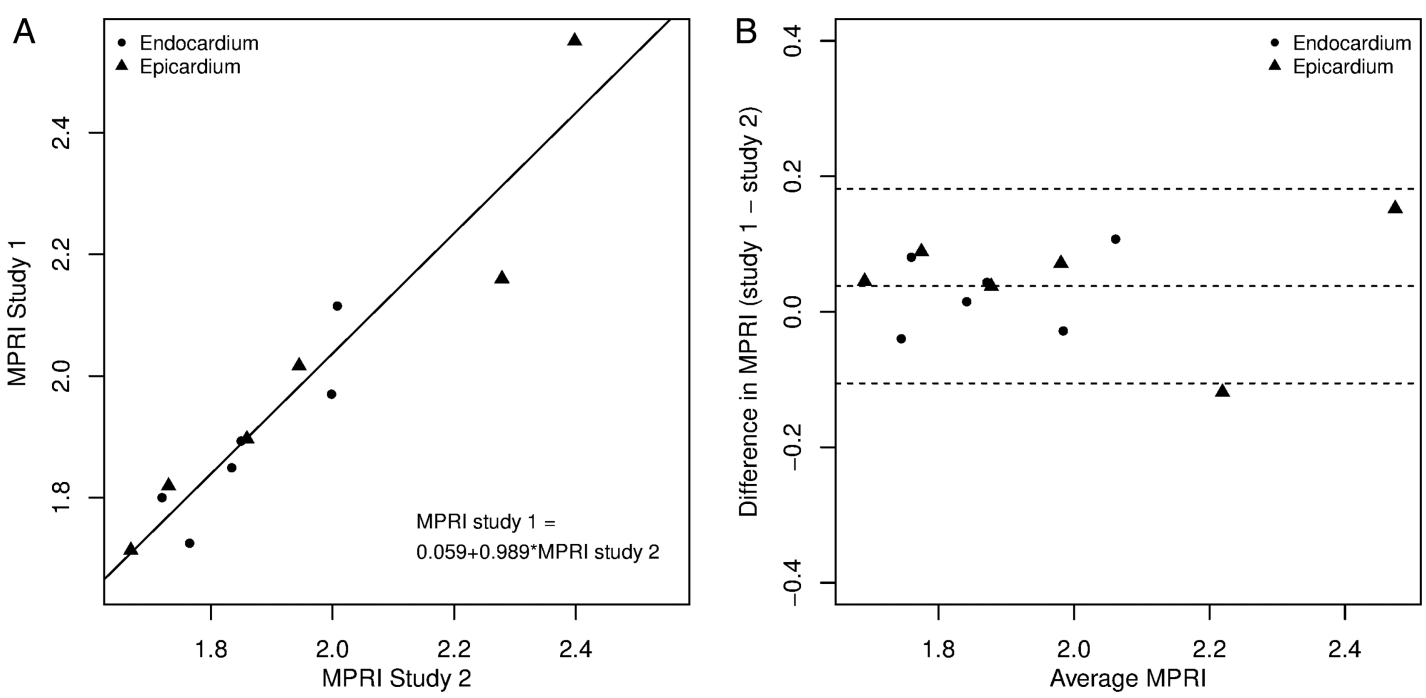

Figure 4 Agreement of Myocardial Perfusion Reserve Index (MPRI) between Study 1 and 2. Linear regression graph (A) and Bland-Altman plot (B) of endocardial (circles) and epicardial (triangles) MPRI.

favourable reproducibility data on a small number of normal participants and patients with coronary artery disease. ${ }^{15-17}$ Elkington et al performed serial adenosine perfusion CMR studies on seven normal participants and nine patients with coronary artery disease. Using a similar normalised upslope analysis, normal participants were found to have a transmural, endocardial and epicardial $\mathrm{CoV}_{2}$ of 39, 37 and $64 \%$, respectively. Patients with coronary artery disease had transmural, endocardial and epicardial $\mathrm{CoV}_{2}$ of 41,50 and $31 \%$, respectively. Chih et al reported their reproducibility findings on serial adenosine CMR studies in 10 'low risk' patients (a presumably near-normal population) and 10 patients with coronary artery disease. Only transmural MPI was calculated in their study. They found a favourable $\mathrm{CoV}_{2}$ for transmural MPI of $18 \%$ in the control participants and patients with coronary artery disease. In a group of 11 young normal participants (mean age $33 \pm 7$ years), Larghat et al, performed a comprehensive analysis of endocardial and epicardial MPI at rest and during adenosine infusion whether measured during diastole or systole. Resting endocardial MPI CoV2 was $17 \%$ measured during systole and 20\% during diastole, whereas stress endocardial MPI CoV2 was $14 \%$ and $15 \%$, respectively. Similar numbers were reported for epicardial MPI. Our measurement of MPI performed equally well with an even lower $\mathrm{CoV}_{2}$ than reported in these prior studies and provides further confirmation that this technique is robust for clinical and research applications.

\section{Limitations}

Several limitations to this study require comment. First, the small sample size may limit the statistical power of the findings. However, despite the small sample size, the reproducibility was excellent and the statistical power was very strong. The sample size we present is comparable to other studies which also showed a high reproducibility of the CMR technique in normal participants and patients with coronary artery disease. ${ }^{15-17}$ Our finding of high reproducibility is in keeping with the excellent reproducibility reported for other CMR parameters, such as LV volume and mass. ${ }^{22}$ Grothues et al found a coefficient of variability of $5.7 \%$ in LV stroke volume across subgroups of normal, heart failure and patients with LV hypertrophy. This high reproducibility translated to a smaller sample size needed to observe changes in the measured parameter (eg, to observe a $10 \mathrm{~mL}$ change in $\mathrm{LV}$ stroke volume, only six patients would need to be studied by CMR vs 37 patients by echocardiography). Second, our interstudy reproducibly compared scans acquired on two different days that were separated by a moderately broad-time range. Prior studies have reported interstudy time intervals averaging between 13 and 7.7 days. ${ }^{15}$ 16 Our time interval is

Table 3 Intraclass correlation coefficient and coefficient of variation for MPI and MPRI

\begin{tabular}{llllll}
\hline & \multicolumn{1}{l}{ ICC (95\% CI) } & Study 1 $\mathbf{C o V}_{\mathbf{1}}(\%)$ & Study $\mathbf{2} \mathbf{C o V}_{\mathbf{1}}(\%)$ & $\mathbf{C o V}_{\mathbf{2}}(\%)$ \\
\hline MPI (resting) & 0.998 & $(0.992$ to 1.00$)$ & 20.2 & 20.3 & 1.4 \\
MPI (hyperaemia) & 0.963 & $(0.842$ to 0.99$)$ & 13.9 & 14.1 & 3.3 \\
MPRI & 0.940 & $(0.794$ to 0.98$)$ & 11.9 & 11.7 & 3.8 \\
\hline
\end{tabular}

CoV, coefficient of variation; ICC, intraclass correlation coefficient; MPI, Myocardial Perfusion Index; MPRI, Myocardial Perfusion Reserve Index. 
comparable to prior studies, if the median interval (14 days) is considered instead of the mean. One patient in our cohort was scanned 150 days between studies due to an unexpected transportation problem. A broad timeinterval range will skew the mean time interval to appear too high in such a small sample size. At casual glance, this may be a limitation. However, it is of interest that there was strong agreement between the two CMR scans, even when separated by 150 days, and suggests that this patient's heart failure treatment was durable and resulted in a lasting beneficial effect on myocardial perfusion. Finally, Elkington et al presented perfusion analysis data using two methods: Fermi deconvolution and normalised upslope (which was the method used in the current study). The Fermi deconvolution method had superior agreement, although the authors concede that the dual bolus protocol used for Fermi deconvolution calculations is more complex to execute than the single bolus protocol used in the normalised upslope technique, and that residual contrast may interfere with the calculations. Christian et $a l^{23}$ also compared the Fermi deconvolution and normalised upslope methods with absolute myocardial blood flow (in $\mathrm{mL} / \mathrm{min} / \mathrm{g}$ ) determined by radiolabeled microspheres in an experimental animal model. Although MPI did not fall on the line of identity with absolute myocardial blood flow, a linear relationship was nevertheless demonstrated between MPI and myocardial blood flow such that a decline in myocardial blood flow resulted in a lower MPI.

\section{CONCLUSIONS}

The semiquantitative evaluation of resting and hyperaemic myocardial perfusion using a normalised upslope analysis during adenosine stress CMR is a highly reproducible technique with strong interstudy agreement in patients with NIDCM. Such reproducibility should provide accurate assessment of changes in MPI resulting from heart failure treatment.

Acknowledgements The authors would like to thank Adam Stein, AS, RT (MRI), Francesca Sabo, BS, RT (MRI), Donald CiFelli, BS, RT (MRI), Barbara Konz, RN, Amber Brock, RN, Debra Rassel, RN, Linda Howerton, RN and Brenda White, RN for their contributions to this study. Gd-DTPA was used off-label for myocardial perfusion imaging.

Contributors MAL participated in the design of the study, verified image analysis, and drafted the manuscript. SPB and DWA enrolled patients, coordinated studies and performed the image analysis. SPB and LW performed the statistical analysis. $\mathrm{HO}$ and DBS contributed to the design of the study. MWK conceived and designed the study and contributed to manuscript preparation. All authors read, provided critical revisions and approved the final manuscript.

Funding This study was supported in part by a Discovery Grant from the Vanderbilt University Medical Center and by the Vanderbilt CTSA grant UL1RR024975 NCRR/NIH. Drs Adkisson and Bell were supported by NIH Training Grant T32 HL 07411-31. The contents are solely the responsibility of the authors and do not necessarily represent official views of the National Center for Advancing Translational Sciences or the National Institutes of Health. This study was supported in part by Clinical and Translational Science
Award UL1RR024975 NCRR/NIH, and Drs Adkisson and Bell were supported by NIH Training Grant T32 HL 07411-31 (Bethesda, MD USA).

Competing interests None.

Ethics approval The study was approved by the Vanderbilt Institutional Review Board (IRB study number 070824) and the Tennessee Valley Department of Veterans Affairs IRB (IRB study number 572).

Provenance and peer review Not commissioned; externally peer reviewed.

Data sharing statement Extra data can be accessed via the Dryad data repository at doi:10.5061/dryad.4j12q.

Open Access This is an Open Access article distributed in accordance with the Creative Commons Attribution Non Commercial (CC BY-NC 4.0) license, which permits others to distribute, remix, adapt, build upon this work noncommercially, and license their derivative works on different terms, provided the original work is properly cited and the use is non-commercial. See: http:// creativecommons.org/licenses/by-nc/4.0/

\section{REFERENCES}

1. Gerber BL, Raman SV, Nayak K, et al. Myocardial first-pass perfusion cardiovascular magnetic resonance: history, theory, and current state of the art. J Cardiovasc Magn Reson 2008;10:18.

2. Schwitter J, Wacker CM, van Roosum AC, et al. MRI-IMPACT: comparison of perfusion-cardiac magnetic resonance with single-photon emission computed tomography for the detection of coronary artery disease in a multicentre, multivendor, randomized trial. Eur Heart J 2008;29:480-9.

3. Schwitter J, Wacker CM, Wilke N, et al. MR-IMPACT II: Magnetic Resonance Imaging for Myocardial Perfusion Assessment in Coronary artery disease Trial: perfusion-cardiac magnetic resonance vs. single-photon emission computed tomography for the detection of coronary artery disease: a comparative multicentre, multivendor trial. Eur Heart J 2013;34:775-81.

4. Greenwood JP, Maredia N, Younger JF, et al. Cardiovascular magnetic resonance and single-photon emission computed tomography for diagnosis of coronary heart disease (CE-MARC): a prospective trial. Lancet 2012;379:435-60. http://dx.doi.org/10.1016/ S0140-6736(11)61335-4

5. Panting JR, Gatehouse PD, Yang GZ, et al. Abnormal subendocardial perfusion in cardiac syndrome $\mathrm{X}$ detected by cardiovascular magnetic resonance imaging. $N$ Engl $J$ Med 2002;346:1948-53.

6. Lanza GA, Buffon A, Sestito A, et al. Relation between stress induced myocardial perfusion defects on cardiovascular magnetic resonance and coronary microvascular dysfunction in patients with cardiac syndrome X. J Am Coll Cardiol 2008;51:466-72.

7. Neglia D, Michelassi C, Trivieri MG, et al. Prognostic role of myocardial blood flow impairment in idiopathic left ventricular dysfunction. Circulation 2002;105:186-93.

8. Neglia D, Parodi O, Gallopin M, et al. Myocardial blood flow response to pacing tachycardia and to dipyridamole infusion in patients with dilated cardiomyopathy without overt heart failure. A quantitative assessment by positron emission tomography. Circulation 1995;92:796-804

9. Vatner SF. Reduced subendocardial myocardial perfusion as one mechanism for congestive heart failure. Am J Cardiol 1988;62:94E-8E.

10. Vatner SF, Shannon R, Hittinger L. Reduced subendocardial coronary reserve. A potential mechanism for impaired diastolic function in the hypertrophied and failing heart. Circulation 1990;81: III8-14.

11. Hittinger L, Shannon RP, Bishop SP, et al. Subendomyocardial exhaustion of blood flow reserve and increased fibrosis in conscious dogs with heart failure. Circ Res 1989;65:971-80.

12. Katz AM. Cardiomyopathy of overload. A major determinant of prognosis in congestive heart failure. $N$ Engl $J$ Med 1990;322:100-10.

13. Neubauer S. The failing heart-an engine out of fuel. $N$ Engl J Med 2007;356:1140-51.

14. Bell SP, Adkisson DW, Ooi $\mathrm{H}$, et al. Impairment of subendocardial perfusion reserve and oxidative metabolism in nonischemic dilated cardiomyopathy. J Card Fail 2013;19:802-10.

15. Elkington AG, Gatehouse PD, Ablitt NA, et al. Interstudy reproducibility of quantitative perfusion cardiovascular magnetic resonance. J Cardiovasc Magn Reson 2005;7:815-22. 
16. Chih S, Macdonald PS, Feneley MP, et al. Reproducibility of adenosine stress cardiovascular magnetic resonance in multi-vessel symptomatic coronary artery disease. J Cardiovasc Magn Reson 2010;12:42.

17. Larghat AM, Maredia N, Biglands J, et al. Reproducibility of first-pass cardiovascular magnetic resonance myocardial perfusion. JMRI 2013;37:865-74.

18. Lauerma K, Virtanen KS, Sipila LM, et al. Multislice MRI in the assessment of myocardial perfusion in patients with single-vessel proximal left anterior descending artery disease before and after revascularisation. Circulation 1997;96:2859-67.

19. Al-Saadi N, Nagel E, Gross M, et al. Noninvasive detection of myocardial ischemia from perfusion reserve based on cardiovascular magnetic resonance. Circulation 2000;101:1379-83.
20. Schwitter J, Nanz D, Kneifel S, et al. Assessment of myocardial perfusion in coronary artery disease by magnetic resonance: a comparison with positron emission tomography and coronary angiography. Circulation 2001;103:2230-5.

21. Lachin JM. The role of measurement reliability in clinical trials. Clin Trials 2004;1:553-66.

22. Grothues F, Smith GC, Moon JCC, et al. Comparison of interstudy reproducibility of cardiovascular magnetic resonance with two-dimensional echocardiography in normal subjects and in patients with heart failure or left ventricular hypertrophy. Am J Cardiol 2002;90:29-34.

23. Christian TF, Rettmann DW, Aletras AH, et al. Absolute myocardial perfusion in canines measured by using dual-bolus first-pass MR imaging. Radiology 2004;232:677-84. 